\title{
A Borehole Muon Telescope for Underground Muography
}

\author{
Luigi Cimmino, ${ }^{1,2}$ Fabio Ambrosino, ${ }^{1,2}$ Antonio Anastasio, ${ }^{2}$ Mariaelena D'Errico, ${ }^{1,2}$ Vincenzo Masone, ${ }^{2}$ \\ Lorenzo Roscilli, ${ }^{2}$ and Giulio Saracino ${ }^{1,2}$ \\ ${ }^{1}$ University of Naples Federico II, Naples, Italy \\ ${ }^{2}$ INFN, Divisioin of Naples, Naples, Italy
}

\begin{abstract}
Radiographic imaging with muons by absorption, also called Muon Radiography or Muography, is a methodology based on the characteristic of the matter to be crossed by high energy muons. This physical property allows muons to pass through the material with a measurable degree of absorption depending on the density of the material. Muon Radiography applies to several different situations and is particularly suitable for investigating subsoil of civil or archaeological interest. This kind of applications needs the muon detector to be installed below the target region. A novel borehole cylindrical detector has been built and tested for use in harsh conditions and for limited space installations. It is based on the past expertise with scintillator detectors and is composed of two types of scintillating elements, bar-shaped and arc-shaped. Due to its size, it can be easily installed in drilled holes of $25 \mathrm{~cm}$ in diameter or more, typically economical to make. Here, we describe the idea, commissioning, and some preliminary results.
\end{abstract}

Keywords: underground muography, radiation detector, applied physics, mining engineering, geological surveys DOI: 10.31526/JAIS.2022.279

\section{INTRODUCTION}

In the muographic field, using compact detectors is advantageous when one wants to carry out direct imaging of underground or buried structures $[1,2,3,4,5,6$, 7]. Unlike Muography applied to volcanoes $[8,9,10,11,12,13,14,15$, 16], where the use of large detectors requires installation in ad hoc structures or in any case in large natural spaces, in underground Muography, the spaces that can be used for the installation of detectors are almost always limited. Examples of these limitations are found almost everywhere in the literature and generally persist even if it is possible to gain space by digging.

For this reason, we designed a cylindrical detector that is well suited to this type of applications, in particular for archaeological and civil purposes. It maximizes the sensitive area allowing to obtain $360^{\circ}$ radiographic imaging despite its small size. Unlike its predecessors $[1,17]$, this cylindrical detector, obtained with plastic scintillators read by Silicon photomultipliers (SiPMs), has the advantage of exploiting arc-shaped scintillating elements.

The detector can be placed on a surface or suspended inside a borehole with a minimum diameter of $25 \mathrm{~cm}$. This type of wells is obtained with conventional and economical drilling techniques, usually deep up to a hundred meters. Furthermore, the device has low power consumption, which allows it to be powered by batteries recharged by solar panels, and the ease of use allows it to be used by technical staff.

\section{PARTS AND CONSTRUCTION OF THE DETECTOR}

The cylindrical detector is constituted by two identical semicylinders. The semicylinder is very simple to assemble and involves the use of a few essential elements and parts that give mechanical stability to the entire detector. This simplifies the production, especially in the perspective of industrial manufacturing.

The elements sensitive to the passage of muons are the plastic scintillators which are in two different shapes, bar and arc, both with a rectangular section, as can be seen in Figure 1. They are arranged to form a semicylindrical surface, with the aid of an assemblable structure that determines the correct positioning of each scintillator, guarantees their optical isolation, and optimizes the transport of light through the scintillating elements.

According to a previous study [18], this optimization is the real strength of the detector design. Each scintillator housing is equipped with small spacers that separate the scintillators from the internal wall. Thus, especially in the case of arc scintillators, a thin layer of air is created which allows the phenomenon of total reflection of the light that propagates inside the scintillator.

The assemblable structure is made of Acrylonitrile Butadiene Styrene (ABS) and is obtained by stacking together four 3D printed rack elements per semicylinder. Scintillator bars are read at both ends by SiPMs. At the bottom ends, 32 SiPMs are housed in a 3D printed part which is used as the base for the semicylinder; a cap is glued to each end at the top and a single SiPM is screwed to each cap. Arc-shaped scintillators are a quarter of a circumference in length and are read at one end only.

Once the semicylinder is assembled, the structure is consolidated by two aluminum bars. They are placed inside the semicylinder and the front-end electronics (FEE) boards are screwed to them. The FEE boards are equipped with the EASIROC chip [19], an 

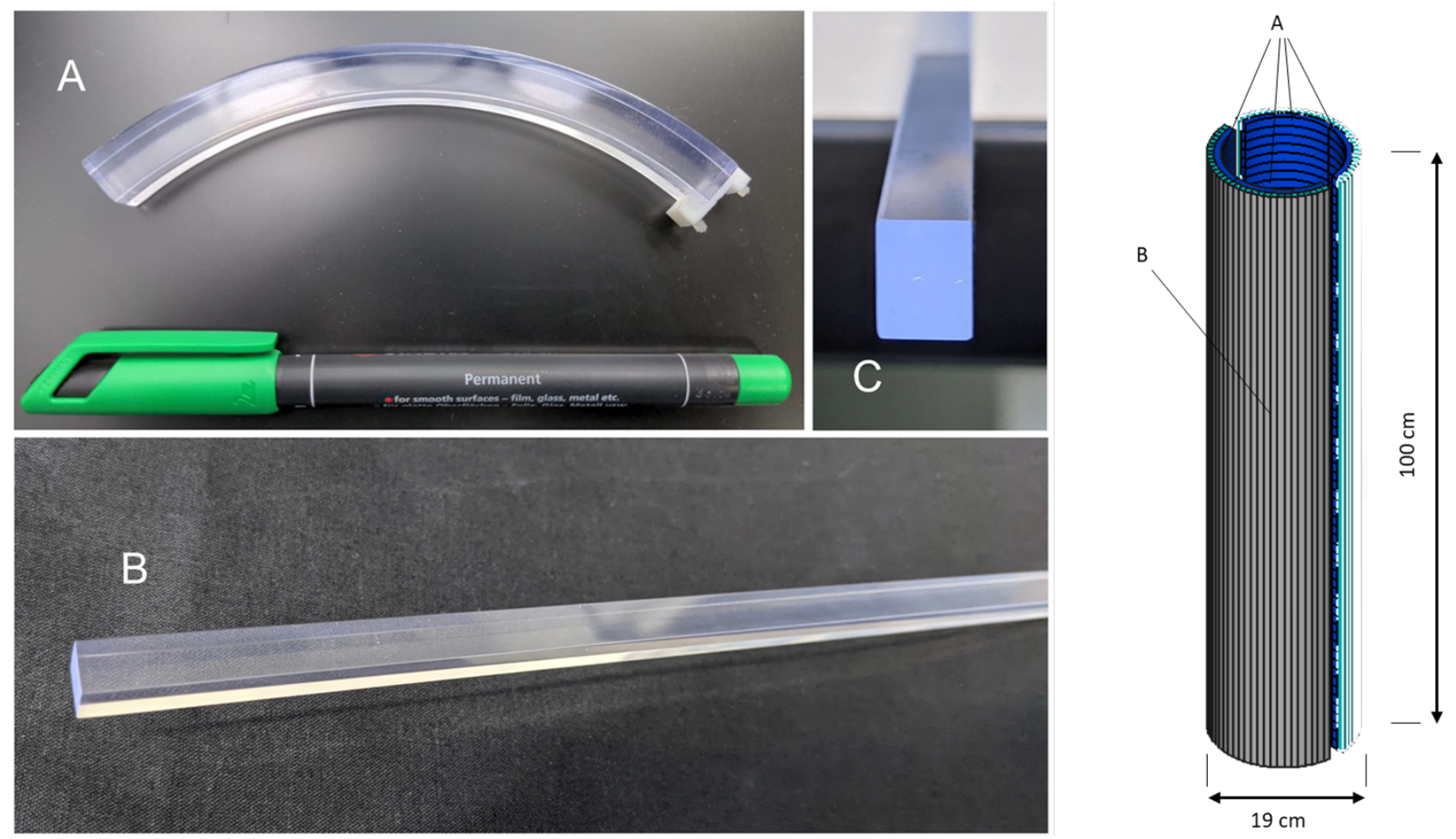

FIGURE 1: (A) Arc-shaped scintillator. (B) $1 \mathrm{~m}$ long scintillator bar. (C) Magnification of the cross section of a bar. On the right, the CAD model of the cylindrical detector.

ASIC that performs the readout of 32 SiPMs, thus measuring the passage of muons through the detector's scintillating elements. The charge collected by each SiPM is sampled and digitized through a slow electronics chain. By programming the FEE boards, one sets the working conditions of the SPMs, hence of the detector. Among all, the main parameters that determine the working conditions of the SiPMs are the high voltage applied to them and the threshold values of signals, produced by SiPMs and processed through a fast electronics chain, so that they are recognized as signals due to the passage of muons.

A Master board sets and reads the 12 FEE boards of the detector. It is embedded with a single-board ARM micro computer, that manages all operations of the detector based on the DAQ system programming. Through a programmable trigger logic, the Master board determines if signals coming from the FEE boards are all due to the passage of a muon through one or both of the semicylinders. The main properties of the DAQ system and the characterization of the front-end electronics are reported in [20].

The detector is finally closed by a stainless steel cylindrical shell which serves to protect it from damage and which guarantees its watertight seal and optical isolation from ambient light sources.

\section{COMMISSIONING AND FIRST USES OF THE DETECTOR}

The detector has been commissioned as reported in [21], and good performance has been achieved, as shown by general stability in the functioning of the detector and in the data acquisition, as shown in the top plot in Figure 3. The characterization and laboratory tests made it possible to set an optimal working point, and the measured muon flux in the open sky with the detector in a vertical position is in good agreement with the muon flux expected under the same condition. On the other hand, the expected cavity detection performance was estimated using a GEANT4 [22] simulation.

The detector has been then deployed in the Bourbon Tunnel inside the Mt. Echia hill in Naples (Italy) to be tested. Starting from May 2021, a series of measurements were carried out from different points of view of the section of the Bourbon Tunnel previously imaged with the MURAY [2] and MIMA [23] detectors.

A further proof of the correct functioning of the detector was given by the comparison between the radiograph of a part of the Bourbon Tunnel obtained from previous observations with the MURAY detector and that obtained with the cylindrical detector. As shown in Figure 3, while not using the same statistic, all the chambers seen with the cylindrical detector are seen by the MURAY detector. The discrepancy between the signals measured by the two detectors is due to the fact that exposure times are different. Furthermore, to emphasize signals measured by the cylindrical detector, we limit the relative transmission to a value that makes some low transmittance regions of the radiograph more visible other than D. Some signals are still missing from the radiograph of the MURAY detector. 

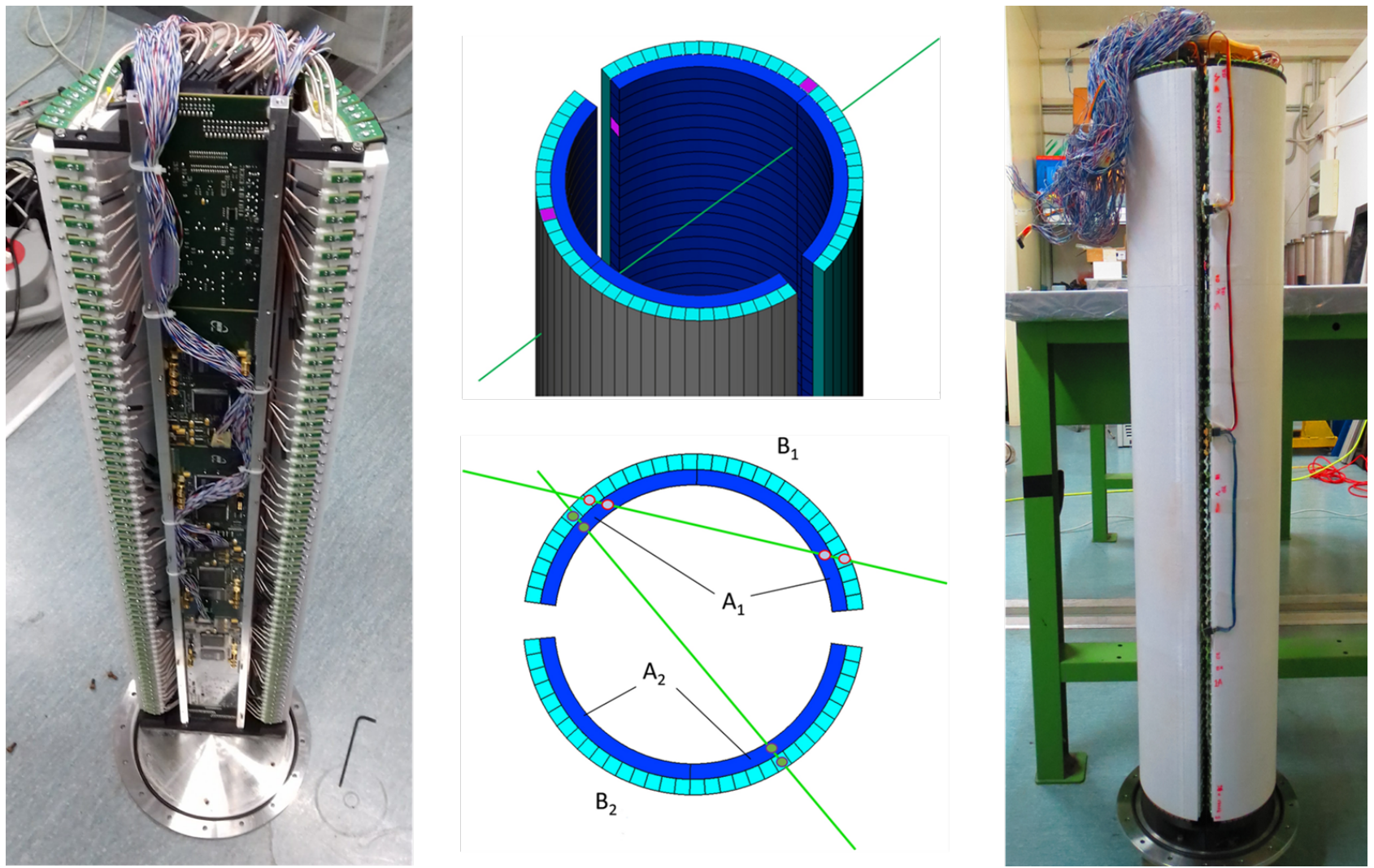

FIGURE 2: Left: assembled semicylinder with electronics. Center: schematic representations of a muon passing through the detector that lights up scintillators (in violet) and of the optimized trigger system which satisfies the logic (A1 and B1) or (A2 and B2). Right: the cylindrical detector.

The purpose of these measurements is to obtain a 3D Muography of the underground region indicated in Figure 3 with the letter $\mathrm{H}$, by applying the 3D reconstruction technique based on the three-dimensional reconstruction of the internal structure of a body using multiple radiographs taken from various angles, developed in [24].

\section{CONCLUSIONS}

A cylindrical muon detector equipped with arc-shaped plastic scintillators has been designed and built. The ease of construction makes it particularly suitable for industrial production. The performances measured during the first tests are very good, and the analysis of the data acquired inside Monte Echia in the city of Naples (Italy) is underway. Great attention was paid to the transport and the detection of the scintillation light in order to achieve the best performance. The cylindrical detector is equipped with a trigger system optimized to obtain a $360^{\circ}$ radiograph. The detector is robust, watertight, and low power consuming for use in harsh environments, and the ease of use makes it particularly suitable for use by technical stuff.

\section{ACKNOWLEDGMENTS}

The realization of the detector prototype was supported by the STRESS Scarl company, in the frame of the project METROPOLIS - PON 03PE_00093_4 (DD MIUR prot. no. 0000791 of the 06/03/2014) funded by the Italian Ministry for Education, University and Research (PON R\&C 2007-2013, DD 713/Ric of the 29/10/2010 Tit III). We are grateful to the technical staff from the Italian National Institute for Nuclear Physics for their support, in particular for the electronics, the mechanical design, and the detector assembly. We are also grateful to the staff of the supercomputing infrastructure SCoPE of the University of Naples Federico II for supporting us in the Monte Carlo simulation campaigns. A special thank goes to Valerio Gentile, Nicola Mori, Pasquale Noli, and Alessandro Varriale for their contributions in the Monte Carlo simulations; to Valentina James, Marilena Esposito, Davide Sala, and Lucio Amato for their support; and to Paolo Strolin. 

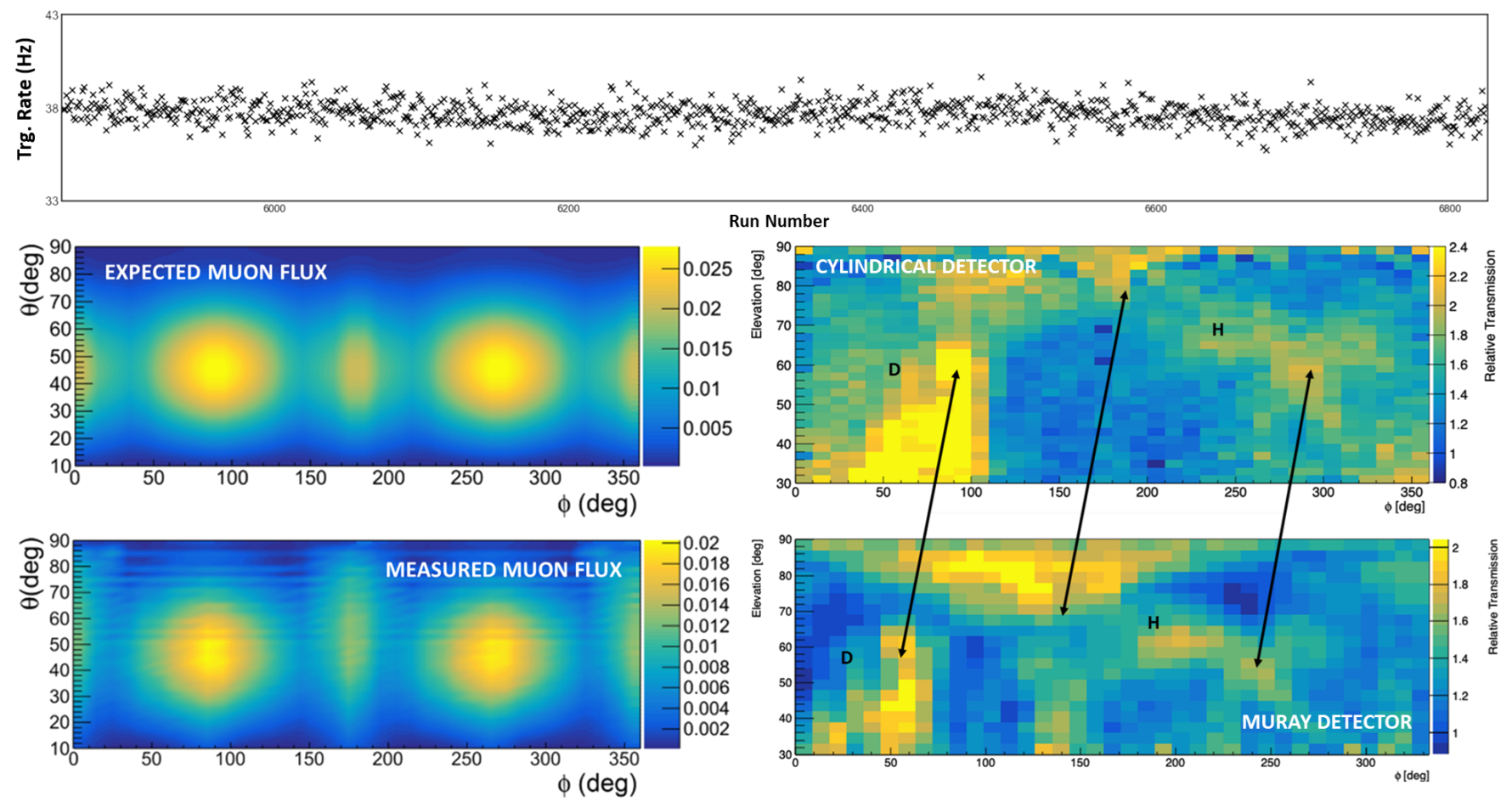

FIGURE 3: Top: trigger rate measured over one week in the open sky with the detector in a horizontal position. Left: comparison between the expected muon flux and the measured muon flux in the open sky with the detector in a vertical position. Right: comparison between the radiography of a part of the Bourbon Tunnel obtained with the cylindrical detector and the MURAY detector.

\section{References}

[1] M. Menichelli, S. Ansoldi, M. Bari, M. Basset, R. Battiston, S. Blasko, F. Coren, E. Fiori, G. Giannini, D. Iugovaz, A. Papi, S. Reia, and G. Scian. A scintillating fibres tracker detector for archaeological applications. Nuclear Instruments and Methods in Physics Research Section A: Accelerators, Spectrometers, Detectors and Associated Equipment, 572(1):262-265, 2007. Frontier Detectors for Frontier Physics.

[2] G. Saracino, L. Amato, F. Ambrosino, G. Antonucci, L. Bonechi, L. Cimmino, L. Consiglio, R. D’Alessandro, E. De Luzio, G. Minin, P. Noli, L. Scognamiglio, P. Strolin, and A. Varriale. Imaging of underground cavities with cosmic-ray muons from observations at mt. echia (naples). Scientific Reports, 7, 2017.

[3] M. D'Errico. Muography applied to archaeology: Search and 3d reconstruction of hidden cavities. Nuovo Cimento della Societa Italiana di Fisica C, 43(4-5), 2020.

[4] G. Baccani, L. Bonechi, M. Bongi, D. Brocchini, N. Casagli, R. Ciaranfi, L. Cimmino, V. Ciulli, R. D’Alessandro, C.D. Ventisette, A. Dini, G. Gigli, S. Gonzi, S. Guideri, L. Lombardi, B. Melon, N. Mori, M. Nocentini, P. Noli, G. Saracino, and L. Viliani. Muon radiography of ancient mines: The san silvestro archaeo-mining park (campiglia marittima, tuscany). Universe, 5, 2019.

[5] L.G. Dedenko, A.K. Managadze, T.M. Roganova, A.V. Bagulya, M.S. Vladimirov, S.G. Zemskova, N.S. Konovalova, N.G. Polukhina, N.I. Starkov, M.M. Chernyavskiy, and V.M. Grachev. Prospects of the study of geological structures by muon radiography based on emulsion track detectors. Bulletin of the Lebedev Physics Institute, 41(8):235-241, 2014.

[6] Z.-X. Zhang, T. Enqvist, M. Holma, and P. Kuusiniemi. Muography and its potential applications to mining and rock engineering. Rock Mechanics and Rock Engineering, 53(11):4893-4907, 2020.

[7] D. Schouten and P. Ledru. Muon tomography applied to a dense uranium deposit at the mcarthur river mine. Journal of Geophysical Research: Solid Earth, 123(10):8637-8652, 2018.

[8] H.K.M. Tanaka, T. Nakano, S. Takahashi, J. Yoshida, M. Takeo, J. Oikawa, T. Ohminato, Y. Aoki, E. Koyama, H. Tsuji, and K. Niwa. High resolution imaging in the inhomogeneous crust with cosmic-ray muon radiography: The density structure below the volcanic crater floor of mt. asama, japan. Earth and Planetary Science Letters, 263(1):104-113, 2007.

[9] C. Cârloganu, V. Niess, S. Béné, E. Busato, P. Dupieux, F. Fehr, P. Gay, D. Miallier, B. Vulpescu, P. Boivin, C. Combaret, P. Labazuy, I. Laktineh, J.-F. Lénat, L. Mirabito, and A. Portal. Towards a muon radiography of the puy de dôme. Geoscientific Instrumentation, Methods and Data Systems, 2(1):55-60, 2013.

[10] P. Noli, F. Ambrosino, L. Bonechi, A. Bross, L. Cimmino, R. D’alessandro, V. Masone, N. Mori, G. Passeggio, A. Pla-Dalmau, G. Saracino, E. Scarlini, and P. Strolin. Muography of the puy de dôme. Ann. Geophys., 60(1), 2017.

[11] L. Oláh, H.K.M. Tanaka, G. Hamar, and D. Varga. Investigation of the limits of high-definition muography for observation of mt sakurajima. Philosophical Transactions of the Royal Society A: Mathematical, Physical and Engineering Sciences, 377(2137):20180135, 2019.

[12] V. Tioukov, A. Alexandrov, C. Bozza, L. Consiglio, N. D'Ambrosio, G. De Lellis, C. De Sio, F. Giudicepietro, G. Macedonio, S. Miyamoto, R. Nishiyama, M. Orazi, R. Peluso, A. Sheshukov, C. Sirignano, S. Maria Stellacci, P. Strolin, and H.K.M. Tanaka. First muography of stromboli volcano. Scientific Report, 9(1):6695, 2019.

[13] M. D'Errico, F. Ambrosino, G. Baccani, L. Bonechi, A. Bross, M. Bongi, A. Caputo, R. Ciaranfi, L. Cimmino, V. Ciulli, R. D'Alessandro, F. Giudicepietro, S. Gonzi, G. Macedonio, V. Masone, B. Melon, N. Mori, P. Noli, M. Orazi, G. Passeggio, R. Peluso, A. Pla-Dalmau, G. Saracino, 
G. Scarpato, L. Scognamiglio, P. Strolin, E. Vertechi, and L. Viliani. Muon radiography applied to volcanoes imaging: the MURAVES experiment at mt. vesuvius. JINST, 15(03):C03014-C03014, 2020.

[14] A. Vesga-Ramirez, D. Sierra Porta, J. Pena Rodriguez, J.D. Sanabria-Gomez, M. Valencia-Otero, C. Sarmiento-Cano, M. Suarez-Duran, H. Asorey, and L.A. Nunez. Muon tomography sites for colombian volcanoes. Ann. Geophys., 63(6), 2020.

[15] H.K.M. Tanaka. Japanese volcanoes visualized with muography. Philosophical Transactions of the Royal Society A: Mathematical, Physical and Engineering Sciences, 377(2137):20180142, 2019.

[16] R. D'Alessandro, F. Ambrosino, G. Baccani, L. Bonechi, M. Bongi, A. Caputo, R. Ciaranfi, L. Cimmino, V. Ciulli, M. D’Errico, F. Giudicepietro, S. Gonzi, G. Macedonio, V. Masone, B. Melon, N. Mori, P. Noli, M. Orazi, P. Passeggio, R. Peluso, G. Saracino, L. Scognamiglio, P. Strolin, E. Vertechi, and L. Viliani. Volcanoes in italy and the role of muon radiography. Philosophical Transactions of the Royal Society A: Mathematical, Physical and Engineering Sciences, 377(2137):20180050, 2019.

[17] M. Basset, S. Ansoldi, M. Bari, R. Battiston, S. Blasko, F. Coren, E. Fiori, G. Giannini, D. Iugovaz, M. Menichelli, S. Reia, and G. Scian. Mgr: An innovative, low-cost and compact cosmic-ray detector. Nuclear Instruments and Methods in Physics Research Section A: Accelerators, Spectrometers, Detectors and Associated Equipment, 567(1):298-301, 2006. Proceedings of the 4th International Conference on New Developments in Photodetection.

[18] L. Consiglio et al. Study of the light response of an arch-shaped scintillator with direct coupling to a silicon photomultiplier readout. Journal of Instrumentation, 14(01), 2019.

[19] S. Callier et al. Easiroc, an easy and versatile readout device for sipm. Physics Procedia, 37:1569-1576, 2012. Proceedings of the 2nd International Conference on Technology and Instrumentation in Particle Physics (TIPP 2011).

[20] L. Cimmino et al. The muraves telescope front-end electronics and data acquisition. Annals of Geophysics, 60(1), 2017.

[21] L. Cimmino, F. Ambrosino, A. Anastasio, M. D’Errico, V. Masone, L. Roscilli, and G. Saracino. A new cylindrical borehole detector for radiographic imaging with muons. Scientific Reports, 11(1), 2021.

[22] S. Agostinelli, J. Allison, K. Amako, J. Apostolakis, H. Araujo, P. Arce, M. Asai, D. Axen, S. Banerjee, G. Barrand, F. Behner, L. Bellagamba, J. Boudreau, L. Broglia, A. Brunengo, H. Burkhardt, S. Chauvie, J. Chuma, R. Chytracek, G. Cooperman, G. Cosmo, P. Degtyarenko, A. Dell'Acqua, G. Depaola, D. Dietrich, R. Enami, A. Feliciello, C. Ferguson, H. Fesefeldt, G. Folger, F. Foppiano, A. Forti, S. Garelli, S. Giani, R. Giannitrapani, D. Gibin, J.J. Gomez Cadenas, I. Gonzalez, G. Gracia Abril, G. Greeniaus, W. Greiner, V. Grichine, A. Grossheim, S. Guatelli, P. Gumplinger, R. Hamatsu, K. Hashimoto, H. Hasui, A. Heikkinen, A. Howard, V. Ivanchenko, A. Johnson, F.W. Jones, J. Kallenbach, N. Kanaya, M. Kawabata, Y. Kawabata, M. Kawaguti, S. Kelner, P. Kent, A. Kimura, T. Kodama, R. Kokoulin, M. Kossov, H. Kurashige, E. Lamanna, T. Lampen, V. Lara, V. Lefebure, F. Lei, M. Liendl, W. Lockman, F. Longo, S. Magni, M. Maire, E. Medernach, K. Minamimoto, P. Mora de Freitas, Y. Morita, K. Murakami, M. Nagamatu, R. Nartallo, P. Nieminen, T. Nishimura, K. Ohtsubo, M. Okamura, S. O'Neale, Y. Oohata, K. Paech, J. Perl, A. Pfeiffer, M.G. Pia, F. Ranjard, A. Rybin, S. Sadilov, E. di Salvo, G. Santin, T. Sasaki, N. Savvas, Y. Sawada, S. Scherer, S. Sei, V. Sirotenko, D. Smith, N. Starkov, H. Stoecker, J. Sulkimo, M. Takahata, S. Tanaka, E. Tcherniaev, E. Safai Tehrani, M. Tropeano, P. Truscott, H. Uno, L. Urban, P. Urban, M. Verderi, A. Walkden, W. Wander, H. Weber, J.P. Wellisch, T. Wenaus, D.C. Williams, D. Wright, T. Yamada, H. Yoshida, and D. Zschiesche. Geant4 - a simulation toolkit. Nuclear Instruments and Methods in Physics Research Section A: Accelerators, Spectrometers, Detectors and Associated Equipment, 506(3):250-303, 2003.

[23] G. Baccani, L. Bonechi, D. Borselli, R. Ciaranfi, L. Cimmino, V. Ciulli, R. D’Alessandro, C. Fratticioli, B. Melon, P. Noli, G. Saracino, and L. Viliani. The mima project. design, construction and performances of a compact hodoscope for muon radiography applications in the context of archaeology and geophysical prospections. Journal of Instrumentation, 13(11), 2018.

[24] L. Cimmino, G. Baccani, P. Noli, L. Amato, F. Ambrosino, L. Bonechi, M. Bongi, V. Ciulli, R. D’Alessandro, M. D’Errico, S. Gonzi, B. Melon G. Minin, G. Saracino, L. Scognamiglio, P. Strolin, and L. Viliani. 3d muography for the search of hidden cavities. Scientific Reports, 9, 2019. 